\title{
Outcomes of minimally invasive sleeve resection
}

\author{
Raul Caso', Thomas J. Watson $^{1,2}$, Puja G. Khaitan ${ }^{2}$, M. Blair Marshall ${ }^{1}$ \\ ${ }^{1}$ Division of Thoracic Surgery, MedStar Georgetown University Hospital, Washington, DC, USA; ${ }^{2}$ Division of Thoracic and Esophageal Surgery, \\ MedStar Washington Hospital Center, Washington, DC, USA \\ Contributions: (I) Conception and design: MB Marshall; (II) Administrative support: TJ Watson; (III) Provision of study materials or patients: MB \\ Marshall; (IV) Collection and assembly of data: R Caso; (V) Data analysis and interpretation: R Caso; (VI) Manuscript writing: All authors; (VII) \\ Final approval of manuscript: All authors. \\ Correspondence to: Raul Caso, MD, MSCI. Department of Surgery, MedStar Georgetown University Hospital, 3800 Reservoir Rd NW, Washington, \\ DC 20007, USA. Email: Raul.Caso@gunet.georgetown.edu.
}

Background: Sleeve resection allows for preservation of lung parenchyma and improved long-term outcomes when compared with pneumonectomy. Little is known about minimally invasive sleeve resection, especially indications, feasibility, technical aspects, complications, and outcomes. We reviewed our institutional experience with sleeve resections via a minimally invasive approach.

Methods: We performed a retrospective review of a prospectively maintained database from 01/01/2010 to 11/01/2017. Indications, operative details, pathology, postoperative complications were reviewed and longerterm follow-up was reviewed.

Results: Fifteen patients were identified ( 5 males, 10 females). Details are presented in Table 1. Patients ranged in age from 7 to 82 years (median, 57 years). Approaches included video-assisted thoracoscopic surgery (VATS) and robotic. Airway sleeve resection was performed in all patients with an additional arterioplasty in 4, one patient having a double sleeve. Length of stay ranged from 3 to 10 days (median, 5 days). Indication for surgery included carcinoid in 5 (1 atypical), NSCLC in 6, and 4 additional pathologies. Complications occurred in 6 patients: air leak [2], pericardial effusion [1], transient brachial plexopathy [1], and atrial fibrillation [2]. There were no anastomotic complications. Median follow-up was 4.2 years. There were no anastomotic strictures.

Conclusions: In experienced centers, sleeve resection via a minimally invasive approach is feasible with acceptable morbidity and mortality. Results in this small series appear comparable with the open approach.

Keywords: Sleeve resection; sleeve lobectomy; bronchoplasty; video-assisted thoracoscopic surgery (VATS); robotic surgery

Submitted Jun 19, 2018. Accepted for publication Sep 20, 2018.

doi: $10.21037 /$ jtd.2018.10.97

View this article at: http://dx.doi.org/10.21037/jtd.2018.10.97

\section{Introduction}

Sleeve resection allows preservation of lung tissue while achieving an oncological resection. Although initially it was intended as a parenchyma-sparing procedure for patients who could not tolerate a pneumonectomy, sleeve lobectomy is now the standard procedure for all anatomically suitable tumors (1). Sleeve procedures result in improved survival, quality of life, reduced loss of lung function, and improved mortality when compared with pneumonectomy (2).
Numerous studies, although mostly retrospective, have shown several advantages of thoracoscopic surgery for major lung resection over thoracotomy (3-6). Recently, a randomized controlled study reported a reduction in postoperative pain and better quality of life with a minimally invasive approach (7). Sleeve lobectomy has traditionally been considered a contraindication to thoracoscopic surgery, however a growing number of series have now reported on the feasibility of video-assisted thoracoscopic surgery (VATS) in sleeve resections (8-13). Thus, the skills 


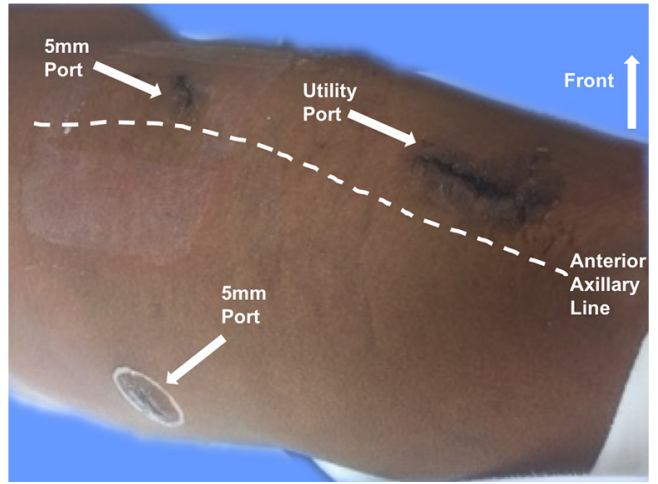

Figure 1 Port placement for a VATS approach. VATS, videoassisted thoracoscopic surgery.

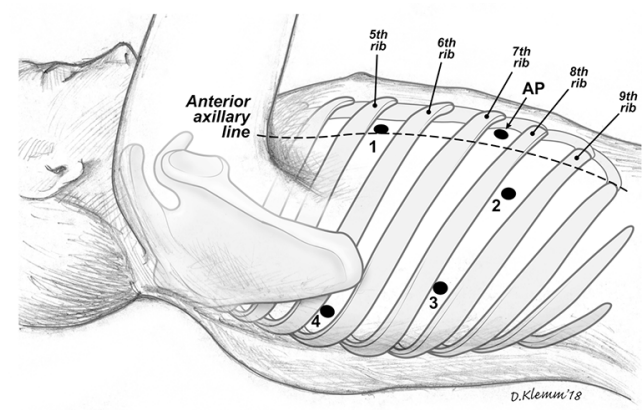

Figure 2 Port placement for a robotic approach. Numbers indicate robotic arm placement: 3 , camera port; AP, $5 \mathrm{~mm}$ assistant port.

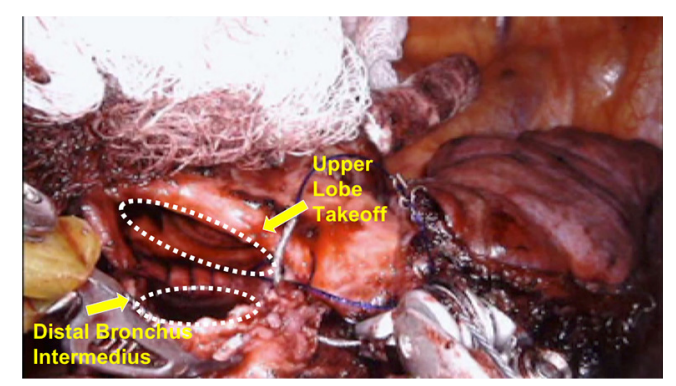

Figure 3 Intraoperative image of airway anastomosis following robotic bronchus intermedius sleeve resection (case 15).

and experience derived from major VATS procedures are being applied to the performance of a sleeve lobectomy with a minimally invasive approach.

Despite the encouraging results, thoracoscopic sleeve resections remain a challenging operation reserved for experienced surgeons. Likely, the steep learning curve associated with this procedure has contributed to the small number of cases reported in the literature. Here we report our outcomes with sleeve resections via a minimally invasive approach.

\section{Methods}

We performed a retrospective review of a prospectively maintained database of patients that underwent thoracoscopic sleeve resections at MedStar Georgetown University Hospital from $01 / 01 / 2010$ to $11 / 01 / 2017$. Indications, operative details, pathology, postoperative complications, and longterm follow-up were reviewed. This study was approved by the MedStar Georgetown University Hospital Institutional Review Board (IRB\# 2012-064).

\section{Operative procedure}

We incorporated two different approaches for our thoracoscopic sleeve resections: VATS and more recently robotic approaches. For the VATS technique, we used the standard 3-port approach and a $5 \mathrm{~mm} \mathrm{30-degree} \mathrm{camera}$ (Figure 1). Two $5 \mathrm{~mm}$ ports, one at the 7 th intercostal space, anterior axillary line, and the 6th intercostal space, posterior axillary line. The utility port was 3.5 to $5 \mathrm{~cm}$ depending on the size of the patient and the body habitus. The position of the utility port was determined after placing the camera. The utility port was placed directly above the pathology to be addressed. Depending on the viewpoint required, the camera was moved between the anterior and posterior ports. For the robotic approach, we utilized a four-port technique with the da Vinci Xi robotic system (Intuitive Surgical, Sunnyvale, CA, USA; Figure 2). A thoracic grasper was used in arm 1, a hook cautery in arm 2, a 30-degree camera in arm 3 and the Maryland forceps in arm 4.

The intercostal muscles were divided in the utility port with camera guidance to facilitate the approach. If no major contraindications were encountered upon initial evaluation, this was followed by mobilization of the lobe, with dissection of the hilar pulmonary lobar branches. Dissection of the airway was completed after division of other hilar structures. Lymphadenectomy was performed typically during mobilization of the airway. Frozen sections of the bronchial margins were obtained before moving on to the bronchoplasty. The airway was then approximated with an end-to-end anastomosis technique using 3-0 or 4-0 PDS (Ethicon, Somerville, NJ, USA), depending on the size of the anastomosis (Figure 3). The 
Table 1 Patient characteristics

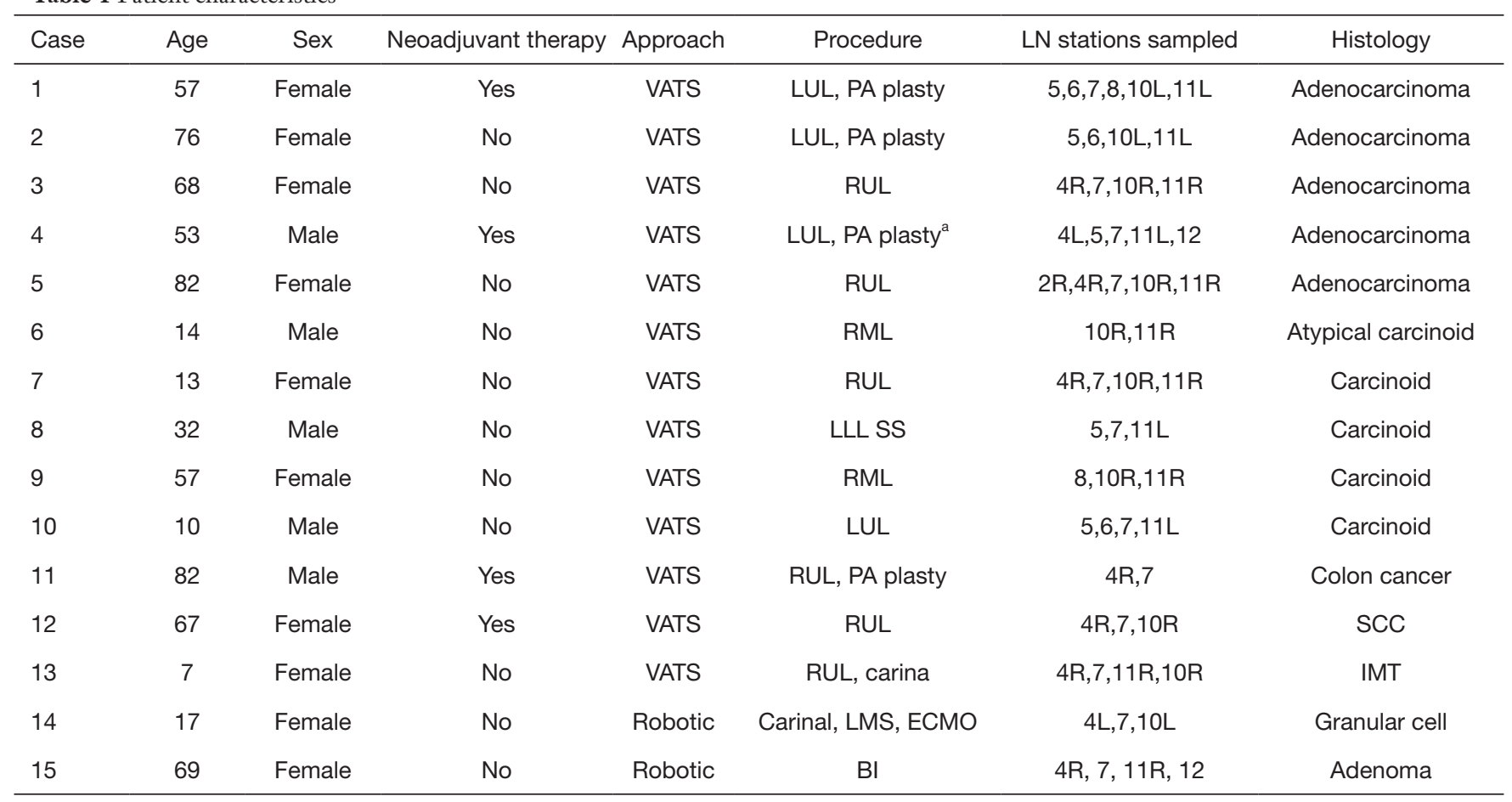

a , patient underwent a double sleeve resection. LN, lymph node; VATS, video-assisted thoracoscopic surgery; LUL, left upper lobe; PA, pulmonary artery; RUL, right upper lobe; RML, right middle lobe; LLL, left lower lobe; SS, superior segmentectomy; LMS, left mainstem; $\mathrm{BI}$, bronchus intermedius; SCC, squamous cell carcinoma; IMT, inflammatory myofibroblastic tumor; ECMO, extracorporeal membrane oxygenation.

vascular anastomosis, when required, was performed following the airway anastomosis. Initially in the series, we harvested a thymic flap or intercostal muscle flap and wrapped it around the airway anastomosis. However, given the added complexity of this step and the lack of true evidence supporting the vascularized pedicle, during the most recent two robotic sleeve resections we did not use a flap (14). At the end of the procedure, intercostal blocks were performed. A single chest tube was left in place at the completion for the majority of procedures. Routine intraoperative bronchoscopy was performed at the completion of each case to evaluate the anastomosis and evacuate any secretions.

\section{Postoperative follow-up}

In the postoperative period, patients were observed in the hospital for a median of 5 days. Chest tubes were pulled prior to discharge. No routine postoperative bronchoscopy was performed. All patients were routinely seen in the office postoperatively.

\section{Results}

Fifteen patients were identified (67\% females). Details are presented in Table 1. Patients ranged in age from 7 to 82 years (median, 57 years). Approaches included 13 VATS and 2 robotic. Airway sleeve resection was performed in all patients. Additional procedures included 4 left upper lobectomies, 1 left lower lobe superior segmentectomy, 5 right upper lobectomies, 2 right middle lobectomies, 1 right upper lobectomy and carinal resection, 1 carinal and left mainstem resection, and 1 bronchus intermedius resection (Figure 3). There were 4 arterioplasties performed, one being a bronchovascular double sleeve (Figure 4). One patient underwent a robotic left hilar release the day before a robotic tracheal and left mainstem resection on extracorporeal membrane oxygenation (ECMO).

The median tumor size was $2.7 \mathrm{~cm}$ (range, 1.1 to $4 \mathrm{~cm}$ ). Pathological examination revealed carcinoid in 5 patients ( 1 atypical), non-small cell lung cancer in 6 , colon cancer in 1 , squamous cell carcinoma in 1 , inflammatory myofibroblastic tumor in 1, granular cell tumor in 1, and bronchial adenoma in 1 . 

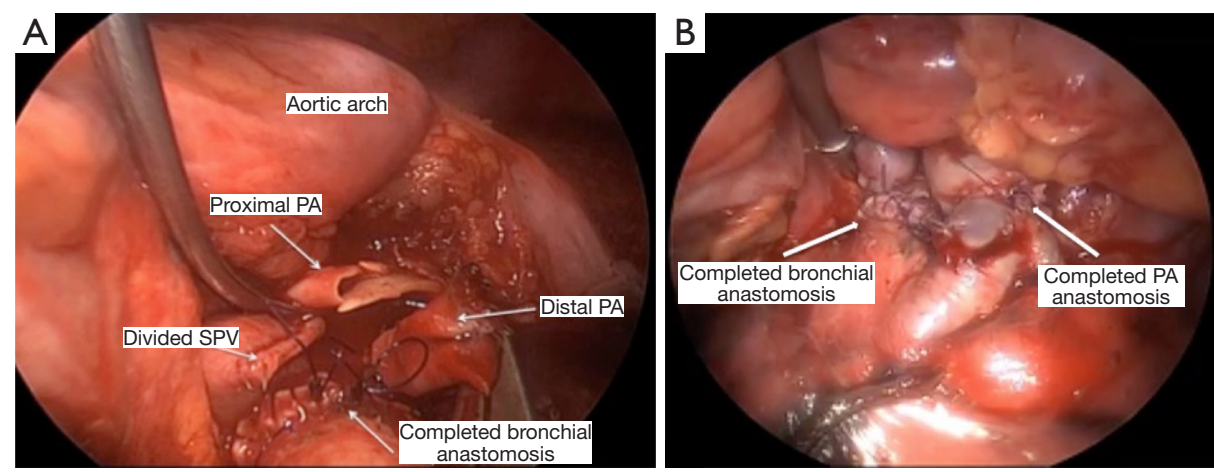

Figure 4 Intraoperative images of a VATS double sleeve resection (case 4) with divided pulmonary artery (A) and completed bronchial and pulmonary artery anastomoses (B). PA, pulmonary artery; SPV, superior pulmonary vein.

Table 2 Postoperative patient characteristics

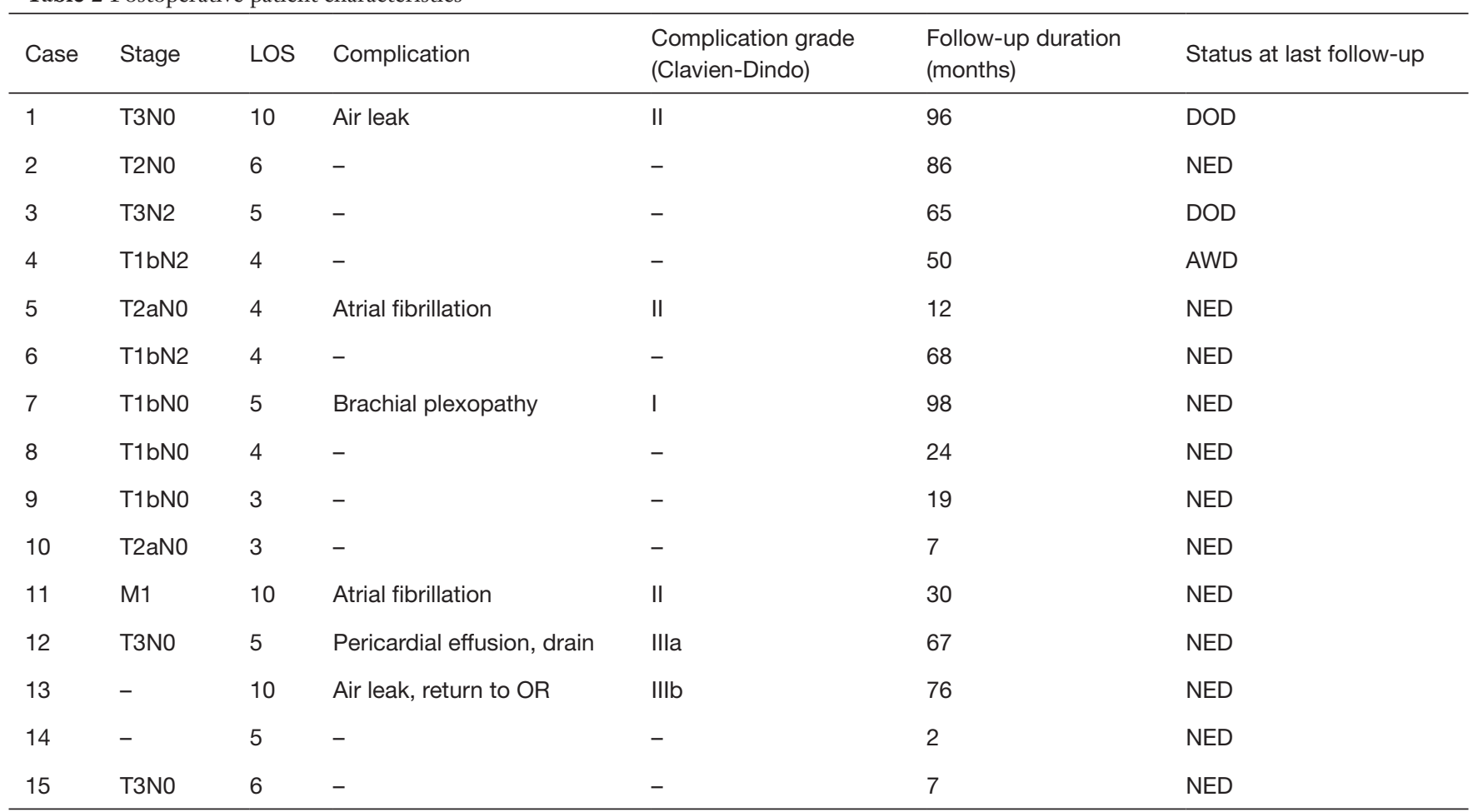

LOS, length of stay; DOD, died of disease; NED, no evidence of disease; AWD, alive with disease.

Length of stay ranged from 3 to 10 days (median, 5 days; Table 2). There were no anastomotic or vascular complications, either short- or long-term. There were no operative mortalities. Nine patients did not have postoperative complications and had uneventful postoperative courses. Six patients had early postoperative complications listed in Table 2. Four (67\%) of the 6 complications were classified as Clavien-Dindo grade I or II (Table 2). One patient was re-admitted to the hospital shortly after being discharged and was found to have a pericardial effusion that required drain placement (ClavienDindo grade IIIa). Her symptoms subsequently resolved and she was discharged home. A second patient, aged 7, had a persistent air leak with fever after a VATS right upper lobe and carinal sleeve resection. She underwent a VATS exploration to evaluate the anastomosis which was 
intact (Clavien-Dindo grade IIIb). She was discharged on postoperative day 10 without further event and is now 13 years old without clinical evidence of stricture. Median follow-up was 4.2 years (range 2 to 98 months) and was complete in all patients. During the follow-up period, two patients died from metastatic non-small cell lung cancer. At last follow-up, one patient was alive with metastatic nonsmall cell lung cancer and 12 patients had no evidence of disease and had no symptoms related to their operation.

\section{Discussion}

Adopting a minimally invasive approach for complex thoracic procedures offers many benefits over traditional thoracotomy, including less pain and quicker recovery without compromising oncologic results (3-6). As a consequence of the increasing experience gained with minimally invasive techniques, technically challenging operations such as sleeve resections are being performed with a thoracoscopic approach. There is ample literature supporting open sleeve lobectomy over pneumonectomy as a result of its improved survival rate, preservation of lung function, and improved operative mortality $(15,16)$. Many patients are candidates for sleeve resection, particularly patients who have a completely atelectatic lobe. In these patients, a sleeve lobectomy should improve their pulmonary function once they have recovered from the operation by eliminating the ventilation perfusion mismatch. A thoracoscopic sleeve resection takes advantage of the improved postoperative outcomes that a minimally invasive approach provides. However, because of the concern about performing an oncologic resection and technical challenges via a minimally invasive approach, this technique has been slow to adopt. Even an open sleeve resection is not widely adopted. Here we report our perioperative and long-term outcomes with minimally invasive sleeve resection.

The surgical principles of a minimally invasive approach to airway sleeve resection are the same as for open surgery. It is critical that the anastomosis of the airway be performed free of tension. Some authors recommend interrupted sutures to allow better size matching, less anastomotic site ischemia and prevent the loosening and entanglement of the sutures (8). Given the technical challenges of the utilization of interrupted sutures through a small utility port, a running suture greatly simplifies the technical challenges of the anastomosis. This was our preferred technique prior to incorporation of a minimally invasive technique.
The advantage of continuous suturing over interrupted suturing for the bronchial anastomosis is the avoidance of multiple sutures exiting the utility port. This can interfere with visualization and performance of the anastomosis. This is of particular importance in minimally invasive thoracic surgery, in which surgeons have a limited field of view and narrow working space (17). In addition, we use an absorbable suture. We have not observed any anastomotic strictures, in particular in children who underwent sleeve anastomosis that are now several years older. Finally, although we routinely buttressed the anastomosis using an intercostal muscle flap, pericardial fat pad or thymic fat pad for our VATS sleeve resections, we did not in the robotic procedures. Given the limitations of the robotic arm positioning and direction, harvesting additional flaps creates new challenges. During the robotic sleeve resections, as we did not observe any intraoperative technical complications related to the performance of the anastomosis, we have adopted the practice of others who do not deem this as a necessary step.

There are few cases describing double sleeve (combined vascular and bronchial) resections with a minimally invasive approach $(11,18-21)$. In a retrospective multicenter study with 13 VATS double sleeve resections for NSCLC, Huang et al. had no conversions to thoracotomy. The median operative time was $263 \mathrm{~min}$ and the median postoperative hospital stay was 10 days (9). Here we report a case of a patient with an adenocarcinoma that underwent neoadjuvant chemotherapy and subsequently we performed a bronchovascular sleeve by VATS. He recovered well postoperatively and went home on day 5 . Double sleeve lobectomy provides an alternative to pneumonectomy in patients presenting with centrally located tumors that involve both the pulmonary artery and bronchus. In patients receiving neoadjuvant treatment, bronchovascular reconstruction is safe and allows for preservation of lung function and rapid recovery (22).

Tracheal resection and carinal reconstruction are a challenging procedure when performed open with high rates of postoperative complications (23). This procedure remains a challenge even in the era of minimally invasive surgery due to the complexity of airway reconstruction and management. Ensuring control of the airway is a potential obstacle when the surgeon is not at the bedside. There are few reports of tracheal resection and carinal reconstruction with a minimally invasive approach due to the complexity of this procedure (13). In our series, 2 patients underwent minimally invasive carinal reconstructions. For one patient, we performed a VATS right upper lobe and carinal sleeve 
resection for an inflammatory myofibroblastic tumor. The second patient with a granular cell tumor involving the carina and left mainstem bronchus underwent carinal and left mainstem resection with a robotic approach under ECMO support. The tracheal defect was closed primarily and the left mainstem was anastomosed to the bronchus intermedius. She recovered well postoperatively and was discharged on postoperative day 5 without complications. Tracheal and carinal resection with a minimally invasive approach may provide the benefits of decreased morbidity and mortality seen with minimally invasive thoracoscopic procedures in general; however careful preoperative planning is critical to a successful operation.

Although this is a small retrospective series, the perioperative outcomes, complications, and length of hospital stay were comparable to that reported by others $(8,23,24)$. Mahtabifard et al. reported four of 13 (31\%) patients undergoing VATS sleeve lobectomy experienced postoperative complications, including atrial fibrillation, anastomotic stricture, reintubation, and bronchial tear requiring repair (8). Wang et al. have described the successful use of VATS sleeve lobectomy with only one patient in their cohort of 15 (6.7\%) experiencing minor complications (25). Such variability likely depends on the characteristics of studied populations, and the small numbers in these series. Zhou et al. retrospectively reviewed their outcomes with VATS versus thoracotomy and found similar complication rates and survival rates despite the surgical approach. The authors did note a longer operative time but a shorter length of hospital stay following VATS sleeve lobectomy as compared to an open approach (26).

\section{Conclusions}

Sleeve resection with a minimally invasive approach can be considered for the management of central airway disease. This appears feasible in select patients, even when arterioplasty or carinal resection is performed. Outcomes in this small series appear comparable to the open approach. It remains unclear if anastomotic healing is improved with a buttress. The magnified view of the anastomosis with the camera during thoracoscopic reconstruction may lead to decreased operative anastomotic complications, however longer follow-up is needed.

\section{Acknowledgements}

None.

\section{Footnote}

Conflicts of Interest: The authors have no conflicts of interest to declare.

Ethical Statement: This study was approved by the MedStar Georgetown University Hospital Institutional Review Board (IRB\# 2012-064).

\section{References}

1. Ma Z, Dong A, Fan J, et al. Does sleeve lobectomy concomitant with or without pulmonary artery reconstruction (double sleeve) have favorable results for non-small cell lung cancer compared with pneumonectomy? A meta-analysis. Eur J Cardiothorac Surg 2007;32:20-8.

2. Stallard J, Loberg A, Dunning J, et al. Is a sleeve lobectomy significantly better than a pneumonectomy? Interact Cardiovasc Thorac Surg 2010;11:660-6.

3. Villamizar NR, Darrabie MD, Burfeind WR, et al. Thoracoscopic lobectomy is associated with lower morbidity compared with thoracotomy. J Thorac Cardiovasc Surg 2009;138:419-25.

4. Swanson SJ, Herndon JE, D'Amico TA, et al. Videoassisted thoracic surgery lobectomy: report of CALGB 39802--a prospective, multi-institution feasibility study. J Clin Oncol 2007;25:4993-7.

5. McKenna RJ, Houck W, Fuller CB. Video-Assisted Thoracic Surgery Lobectomy: Experience With 1,100 Cases. Ann Thorac Surg 2006;81:421-5.

6. Kaseda S, Aoki T, Hangai N, et al. Better pulmonary function and prognosis with video-assisted thoracic surgery than with thoracotomy. Ann Thorac Surg 2000;70:1644-6.

7. Bendixen M, Jørgensen OD, Kronborg C, et al. Postoperative pain and quality of life after lobectomy via video-assisted thoracoscopic surgery or anterolateral thoracotomy for early stage lung cancer: a randomised controlled trial. Lancet Oncol 2016;17:836-44.

8. Mahtabifard A, Fuller CB, McKenna RJ. Video-Assisted Thoracic Surgery Sleeve Lobectomy: A Case Series. Ann Thorac Surg 2008;85:S729-32.

9. Huang J, Li J, Qiu Y, et al. Thoracoscopic double sleeve lobectomy in 13 patients: a series report from multicenters. J Thorac Dis 2015;7:834-42.

10. Gonzalez-Rivas D, Fernandez R, Fieira E, et al. Uniportal video-assisted thoracoscopic bronchial sleeve lobectomy: First report. J Thorac Cardiovasc Surg 2013;145:1676-7. 
11. Gonzalez-Rivas D, Delgado M, Fieira E, et al. Double sleeve uniportal video-assisted thoracoscopic lobectomy for non-small cell lung cancer. Ann Cardiothorac Surg 2014;3:E2.

12. Bertolaccini L, Viti A, Terzi A. Moving beyond the boundary: the emerging role of video-assisted thoracic surgery for bronchoplastic resections. J Thorac Dis 2014;6:1170-2.

13. He J, Wang W, Li J, et al. Video-assisted thoracoscopic surgery tracheal resection and carinal reconstruction for tracheal adenoid cystic carcinoma. J Thorac Dis 2016;8:198-203.

14. Storelli E, Tutic M, Kestenholz P, et al. Sleeve resections with unprotected bronchial anastomoses are safe even after neoadjuvant therapy. Eur J Cardiothorac Surg 2012;42:77-81.

15. Rendina EA, De Giacomo T, Venuta F, et al. Lung conservation techniques: bronchial sleeve resection and reconstruction of the pulmonary artery. Semin Surg Oncol 2000;18:165-72.

16. Burfeind WR, D'Amico TA, Toloza EM, et al. Low morbidity and mortality for bronchoplastic procedures with and without induction therapy. Ann Thorac Surg 2005;80:418-21; discussion 422.

17. Nakagawa T, Chiba N, Ueda Y, et al. Clinical experience of sleeve lobectomy with bronchoplasty using a continuous absorbable barbed suture. Gen Thorac Cardiovasc Surg 2015;63:640-3.

18. Yu DP, Han Y, Zhao QY, et al. Pulmonary lobectomy

Cite this article as: Caso R, Watson TJ, Khaitan PG, Marshall MB. Outcomes of minimally invasive sleeve resection. J Thorac Dis 2018;10(12):6653-6659. doi: 10.21037/jtd.2018.10.97 combined with pulmonary arterioplasty by complete videoassisted thoracic surgery in patients with lung cancer. Asian Pac J Cancer Prev 2013;14:6061-4.

19. Nakanishi R, Yamashita T, Oka S. Initial experience of video-assisted thoracic surgery lobectomy with partial removal of the pulmonary artery. Interact Cardiovasc Thorac Surg 2008;7:996-1000.

20. Liu L, Mei J, Pu Q, et al. Thoracoscopic bronchovascular double sleeve lobectomy for non-small-cell lung cancer. Eur J Cardiothorac Surg 2014;46:493-5.

21. Han Y, Zhou S, Yu D, et al. Video-assisted thoracic surgery (VATS) left upper sleeve lobectomy with partial pulmonary artery resection. J Thorac Dis 2013;5 Suppl 3:S301-3.

22. Maurizi G, D’Andrilli A, Anile M, et al. Sleeve Lobectomy Compared with Pneumonectomy after Induction Therapy for Non-Small-Cell Lung Cancer. J Thorac Oncol 2013;8:637-43.

23. Mitchell JD, Mathisen DJ, Wright CD, et al. Clinical experience with carinal resection. J Thorac Cardiovasc Surg 1999;117:39-52; discussion 52-3.

24. Agasthian T. Initial experience with video-assisted thoracoscopic bronchoplasty†. Eur J Cardiothorac Surg 2013;44:616-23.

25. Li Y, Wang J. Video-assisted thoracoscopic surgery sleeve lobectomy with bronchoplasty: an improved operative technique. Eur J Cardiothorac Surg 2013;44:1108-12.

26. Zhou S, Pei G, Han Y, et al. Sleeve lobectomy by videoassisted thoracic surgery versus thoracotomy for non-small cell lung cancer. J Cardiothorac Surg 2015;10:116. 